\title{
CORRIGENDUM
}

\section{Expression of human PTPN22 alleles}

\author{
C Nielsen, T Barington, S Husby and ST Lillevang \\ Genes and Immunity (2007) 8, 366-367; doi:10.1038/sj.gene.6364397
}

Correction to: Genes and Immunity (2006) E-pub ahead of print: June 1, 2006; doi:10.1038/sj.gene.6364369

Since the publication of the above article, the authors have identified errors in the results and figures.

Please find below the detailed description of the new results and figures in their entirety.

In that work the expression of PTPN22 alleles in individuals heterozygous for the C1858T SNP was measured using a fluorescent PCR-RFLP analysis. By mistake one of the primers used for both the PCR-RFLP and RT-PCR analysis is situated in an intron. Because the non-RT controls excluded contamination with genomic DNA, it is most likely that the published relative expression of PTPN22 alleles was at the pre-mRNA level and not the mRNA level as stated in the paper. All samples have now been reanalyzed with the mRNAspecific primers listed below, and essentially identical results are obtained. The conclusions drawn in the manuscript are therefore valid for both pre-mRNA and mRNA.

RFLP analysis: This was performed using a forward primer: 5'-CTGTACTAGCAACTGCTCCA-3' (corresponding to positions 1891-1910 of the PTPN22 mRNA, GenBank accession no. NM-015967) and a reverse primer: 5'-GGTCTAAGTATCACAGAGTC-3' (corresponding to positions 2088-2107 of the PTPN22 mRNA, GenBank accession no. NM-015967) labeled at its $5^{\prime}$ end with the fluorescent dye FAM (6-carboxyfluorescein) and designed to amplify a $217 \mathrm{bp}$ FAM-labeled mRNAspecific fragment of PTPN22 containing the C1858T SNP. The relative expression of the PTPN22 1858T and 1858C allele was calculated as the ratio of the peak areas of a $217 \mathrm{bp}$ fragment and a $162 \mathrm{bp}$ cleaved fragment.

RT-PCR: This was performed using forward primer 5'-CTGTACTAGCAACTGCTCCA-3' (corresponding to positions 1891-1910 of the PTPN22 mRNA, GenBank accession no. NM-015967) and reverse primer 5'-TCCAGCTTCCTCAACCACAA-3' (corresponding to positions 1966-1985 of the PTPN22 mRNA, GenBank accession no. NM-015967).

\section{Detailed description of the new results and figures}

For the fluorescent capillary electrophoresis, the PCR was performed using a forward primer (5'-CTGTAC TAGCAACTGCTCCA-3' (corresponding to positions 1891-1910 of the PTPN22 mRNA, GenBank accession no. NM-015967) and a reverse primer: 5'-GGTCTAAGTATCACAGAGTC-3' (corresponding to positions 2088-2107 of the PTPN22 mRNA, GenBank accession no. NM-015967)) labeled at its $5^{\prime}$ end with the fluorescent dye FAM and designed to amplify a 217 bp FAM-labeled mRNA-specific fragment of PTPN22 containing the C1858T SNP.

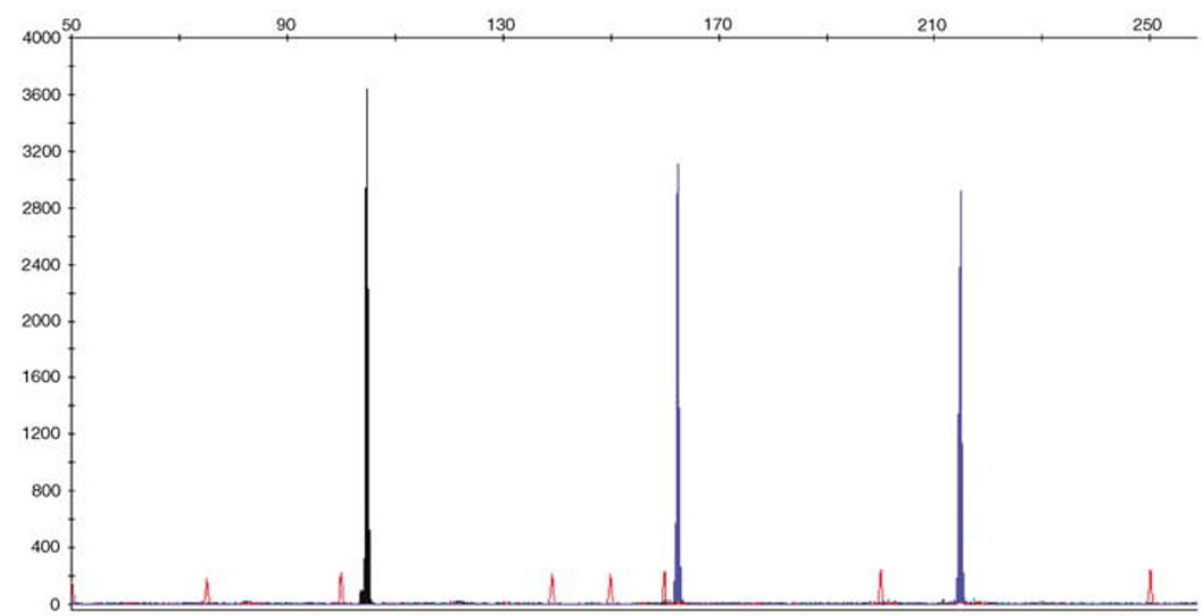

Figure 1 Fragment length analysis by capillary electrophoresis of PTPN22 PCR products. NED- and FAM-coupled fragments are shown in black and blue, respectively. Cleavage reaction products of a C1858T heterozygous sample. Absence of a NED-coupled $148 \mathrm{bp}$ fragment and presence of a NED-coupled $104 \mathrm{bp}$ fragment demonstrates complete cutting by the RsaI endonuclease. 


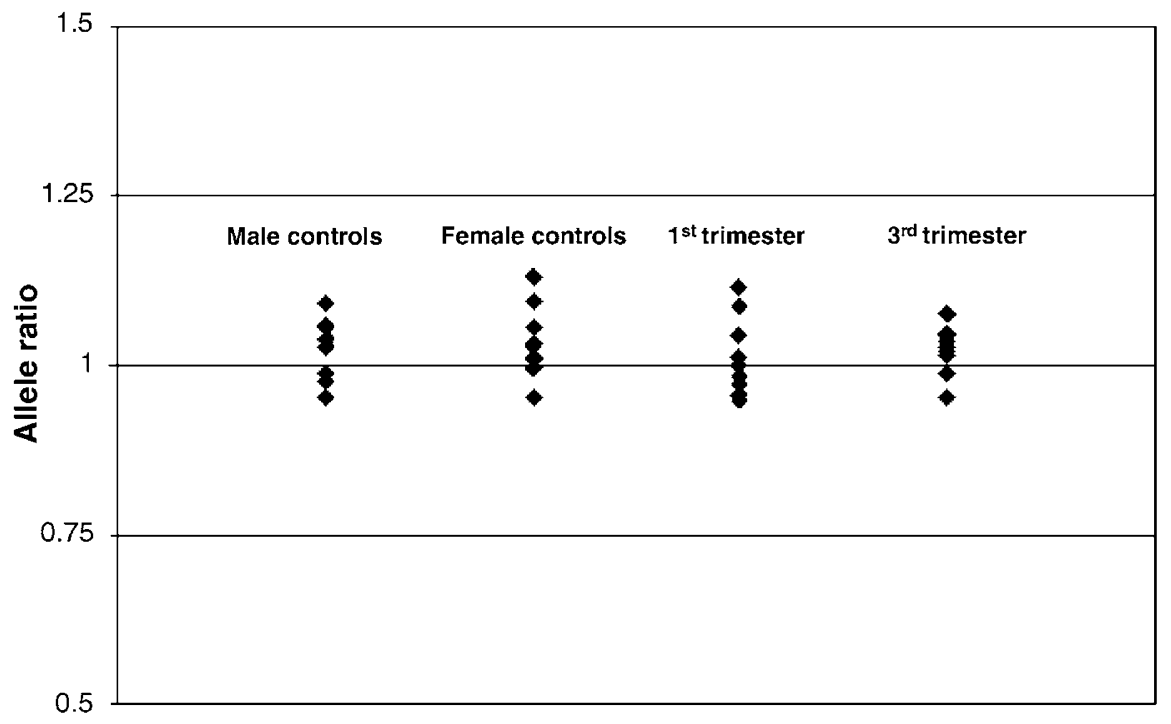

Figure 2 cDNA ratios assayed at the PTPN22 1858C/T SNP. Data are expressed $(n=10-12)$ as the ratio of T:C alleles. The data are the peak area averages of two measurements for each individual sample. There was no significant difference in the allele ratio between the different groups.

\section{Relative allelic expression analysis}

For each cDNA sample from heterozygous individuals, the relative expression of the PTPN22 1858T and 1858C allele was calculated as the ratio of the peak areas of the $217 \mathrm{bp}$ fragment and the $162 \mathrm{bp}$ fragment.

Regulation of PTPN22 $m R N A$ and relative allelic expression in stimulated PBMCs over time

The reaction was carried out with the SYBR Green PCR Master Mix, $3 \mathrm{ml}$ of cDNA and 5 pmol of each mRNAspecific primer (forward primer: 5'-CTGTACTAG CAACTGCTCCA-3' (corresponding to positions 18911910 of the PTPN22 mRNA, GenBank accession no. NM015967); reverse primer: 5'-TCCAGCTTCCTCAACCA CAA-3' (corresponding to positions 1966-1985 of the PTPN22 mRNA, GenBank accession no. NM-015967)) designed to amplify a $95 \mathrm{bp}$ mRNA-specific fragment of PTPN22.

The mRNA induction index of PTPN22 in PBMCs increased approximately twofold following $6 \mathrm{~h}$ of stimulation $(P=0.012$; one-way repeated measures ANOVA; Bonferroni-adjusted $P$-value) and remained relatively unchanged until $48 \mathrm{~h}$ of anti-CD3 and anti-CD28 stimulation, where the mRNA expression of PTPN22 was upregulated approximately eight times compared to non-stimulated cells $(P<0.0001)$. Following $72 \mathrm{~h}$ of stimulation, the mRNA expression of PTPN22 was further upregulated compared to $48 \mathrm{~h}$ of stimulation $(P<0.0001)$ and reached an approximately 24 times higher level compared to nonstimulated PBMCs $(P<0.0001)$. The level of DNA contamination was low as no-reverse transcription controls always resulted in CT values higher than the 40 cycles detected in the RT-PCR (Figures 1-3).

\section{Conclusion}

As published in the article, the expression of PTPN22 $1858 \mathrm{C}$ and $\mathrm{T}$ alleles in anti-CD3- and anti-CD28stimulated PBMCs was increased to the same extent
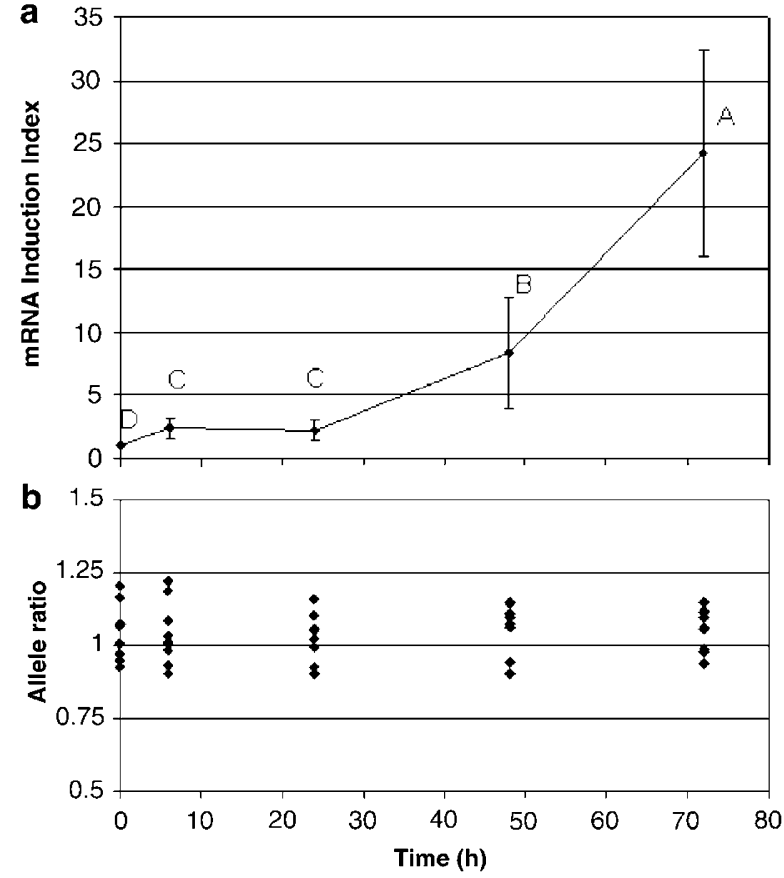

Figure 3 (a) Changes in the mRNA induction index of PTPN22 in PBMCs stimulated with anti-CD3 and anti-CD28 over time $(n=10)$. Data are shown as mean \pm s.d. and values with shared letters are not significantly different $(P>0.05)$. (b) Allele-specific expression assayed at the PTPN22 1858C/T SNP in the same anti-CD3 and anti-CD28 over time. Data are expressed $(n=10)$ as the ratio of T:C alleles. The data are the peak area averages of two measurements for each individual sample. There was no significant difference in the allele ratio between the different stimulation times.

and followed the same kinetics using the new mRNAspecific primers - just at a higher level. The use of the new mRNA-specific primers supported the published absence of any significant sex-specific variability in relative mRNA allele ratio.

The authors would like to apologize for these errors. 